\title{
Using Hybrid Evolutionary Algorithm based Adaptive Filtering
}

\author{
Adnan Alrabea \\ Al-Balqa Applied University \\ Al-Salt, Jordan \\ dr.alrabea@bau.edu.jo
}

\begin{abstract}
Noise degrades the overall efficiency of the data transmission in the networking models which is no different in Cognitive Radio Adhoc Networks (CRAHNs). For efficient opportunistic routing in CRAHN, the Modified SMOR (MSMOR) and Sparsity based Distributed Spectrum Map MSMOR (SDS-M-SMOR) have been developed which provide significant improvement in the overall routing behavior. However, the increase in the noises is inevitable especially in large scale networks which Swarm Optimization (PSO) and Genetic Algorithm (GA) together termed as HPSOGA. The proposed HPSOGA based adaptive filter readjusts the filter constraints in accordance to the channel and the signals, thus mitigates the noise in the reconfigurable systems, like CRAHNs. The key benefit of the HPSOGA based adaptive filter is the global optimization when compared to other, the proposed model with noise cancellation has better performance values than other routing models.
\end{abstract}

Keywords-Cognitive radio adhoc networks; distributed spectrum map; swarm optimization; genetic algorithm

\section{INTRODUCTION}

Data integrity is affected in communication networks by noise elements. Noise incorporates bad performance, noise establishing from framework non-linearity in the wireless anterior end, and impedance between co-positioned wireless nodes inside a network [1]-[3]. To de-noise from the signals, channels are utilized in transmission frameworks. These channels are constructed utilizing hardware segments, which prompts expensive and massive frameworks that can just channel particular bandwidths [4]. However, future communication innovations will have reconfigurable structure and will empower progressed digital communication. CRAHN is one such reconfigurable system that requires adaptive model of noise cancellation according to the channels and the signals. In CRAHNs if the primary users involve the band and there is lot of noise produced from the condition, the sensor does likewise activity of moving endlessly and sensing for different bands; in the event that the sensor choose to utilize that band or not relying upon the outcome.

Adaptive filtering, Evolutionary algorithm, Particle Swarm Optimization, Genetic Algorithm, Peak-to-Signal Noise Ratio, Mean Square Error optimization techniques - by utilizing the proposed noise cancellation model, the noises are eliminated which in turn enhances the opportunistic routing performance. The experimental results show that catalyst the routing degradation through noisy data transmission. In order to resolve this issue, adaptive filtering concepts are mostly used in cognitive radio networks. This paper aims at developing novel noise cancellation system to be coupled with the opportunistic routing model so that the routing as well as transmission performance can be improved. For this purpose, an adaptive filtering technique is developed using hybrid of Particle could separate whether the involved signal is noise or impedance signal then the sensor can either. As the noise is also responsible for the quality of the data transmission, the routing performance is linked with it. Hence in this article, the SDS-MSMOR routing model [15] incorporates a noise cancellation system. As CRAHN is a reconfigurable system, the incorporated noise cancellation must be adaptive and hence the adaptive filter is imposed. This article develops a new adaptive filter based on the HPSOGA to provide global optimal solution for the filter parameters. Unlike other filters which provide only local optimum solutions, this proposed Adaptive filtering enabled SDS-M-SMOR (AF-SDS-M-SMOR) model eliminates the noises more effectively and enhances the opportunistic routing. The rest of the article is prearranged as: Section 2 defines some of the recent research works related to the study. Section 3 explains the proposed system model followed by the adaptive filtering based routing model in Section 4 while Section 5 evaluates its performance. Section 6 makes a conclusion of the research model developed in this article.

\section{RELATED WORKS}

The primary solution for noise cancellation is the silencer systems [5]; though the restrictions like size, price and me agree performance in contrast to lesser frequencies encouraged academics to find alternatives. As a solution, the Active Noise cancellation (ANC) system originated. Most ANC used adaptive FIR filters and LMS [6] \& NLMS [7] owing to their easiness and intrinsic stability. However the negative gradient problem of these techniques led to way for adaptive filters based on evolutionary algorithms. Evolutionary algorithms appeared in [8] to be a compelling possibility to mitigate nearby minima and Eigen value uniqueness issues of conventional ANC plans. The GA, a standout evolutionary computing algorithms, utilized to prepare the weights of a versatile IIR in [9]. GA has been recently connected to dynamic commotion and vibration control [10], demonstrating the viability of the plan by an exploratory investigation. The utilization of GA has been reached out to Voltaire-based nonlinear ANCs in [11]. Each versatile weight of the controller is encoded as a binary string, which is haphazardly allocated toward the beginning of the ANC operation. Utilizing 
reduction of mean-square inaccuracy, the weights are adjusted utilizing GA [12], which utilizes an arrangement of reproduction, crossover, and mutation processes. An ANC conspire in light of a versatile GA has likewise been as of late recommended that fuses versatile possibility for crossover and mutation, and executed in a constant purpose [13]. PSO rose as an appropriate substitution for GA, inferable from its speedier meeting. The computational multifaceted nature of PSO-based plans is additionally not as much as that of GA-based plans that utilize binary coding. From the literature, it has been found that the PSO based adaptive filter is highly efficient than GA and hence this paper explores the idea of utilizing a hybrid evolutionary algorithm based adaptive filter.

\section{SYSTEM MODEL}

In the proposed model, it is expected that a CRAHN users can tune its radio transceiver to any of the permitted channels in the authorized (primary) band. Fig. 1 shows the proposed model of opportunistic routing. A cognitive radio network comprising of $\mathrm{M}$ prime users and $\mathrm{N}$ secondary users is considered. Prime users seize authorize for particular spectrum bands, and be able to involve one's allocated bit of the spectrum. Secondary users don't have allowed spectrum and shrewdly transmit their information by using inert parts of the principal spectrum. Every node has a solitary radio and there is a basic channel coordination instrument at the connection layer that enables neighboring nodes to participate in pair wise communication. The PU action is displayed by the option exponential ON-OFF model. In this model, every PU has two option states: ON and OFF. An ON (occupied) state speaks to the period in which the primary band is involved by PU, while an OFF (idle) state speaks to the period in which the primary band is sit still and can be utilized by CR units. The degree is constrained to the steering convention for CRAHN networks.

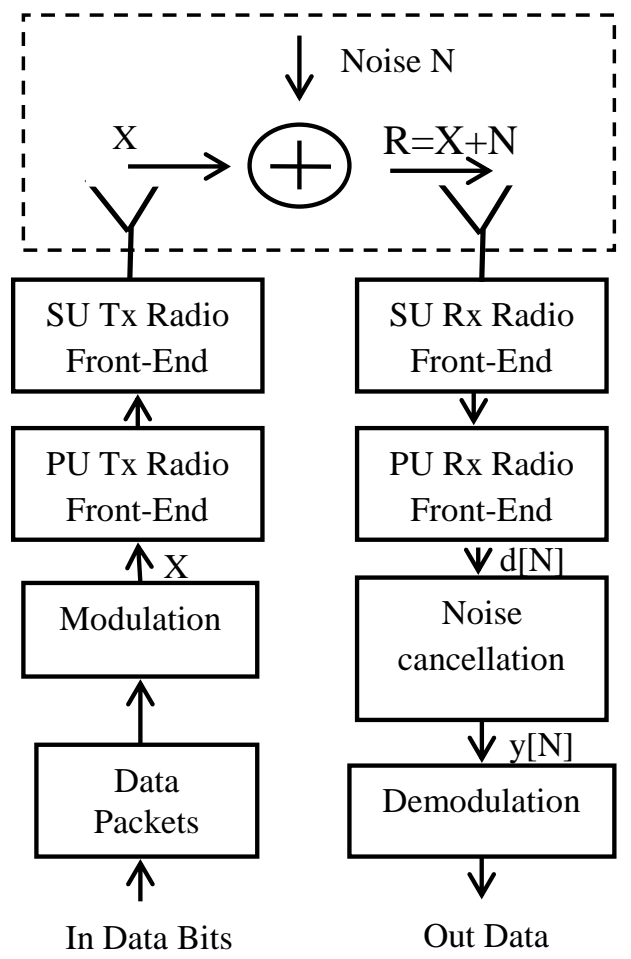

Fig. 1. Proposed model with noise cancellation.

It is expected that the source node is at first aware of the area of the destination, however the last may periodically send position refreshes once the course is in operation. Every node is additionally aware of its own area. There are no earlier presumptions of the number, areas, transmission standard or convention that is trailed by the PU. The proposed model named as AF-SDS-M-SMOR is explained in the following section.

\section{AF-SDS-M-SMOR USING HYBRID PSO-GA (HPSOGA) BASED ADAPTIVE FILTERING}

The theory of an adaptive filter is the time altering, selfregulating features. The inaccuracy signal is computed from the signal flow map of the optimal function. In order to obtain this near optimal solutions, the optimization based filters have been developed. However, the developed filters were gradientdescent based filters which have negative gradient impact and also the solutions were only local optimum. Hence the evolutionary algorithms based adaptive filters were developed, yet through extensive research it has been found that hybrid models of evolutionary algorithms provide improved noise cancellation and in turn enhancing the routing behavior.

HPSOGA algorithm can be depicted as in the following algorithm based on the standard PSO [14] and GA algorithms. Initially put the population size $\mathrm{P}$, acceleration constant $\mathrm{c} 1$ and $\mathrm{c} 2$, crossover probability Pc, mutation probability $\mathrm{Pm}$, partition numberpart $_{\mathrm{n}}$, number of variables in each partition $\mathrm{m}$, number of solutions in each partition $\mathrm{g}$ and the maximum number of iterations $\mathrm{Max}_{\mathrm{itr}}$. Then the process begins as follows (Algorithm 1):

\begin{tabular}{|c|}
\hline Algorithm 1: HPSOGA algorithm \\
\hline Begin \\
\hline Set $\mathbf{t}:=\mathbf{0} / /$ Counter initialization \\
\hline For $(\mathbf{i}=\mathbf{1}: \mathbf{i} \leq \mathbf{P})$ do \\
\hline create an primary population $\overline{\mathbf{X}_{\mathbf{I}}(\mathbf{t}) \text { randomly }}$ \\
\hline calculate the fitness utility of each search solution $\mathbf{f}\left(\overrightarrow{\mathbf{X}_{1}}\right)$ \\
\hline End for \\
\hline Repeat \\
\hline Apply the standard PSO on $\mathbf{X}_{\mathbf{I}}(\mathbf{t})$ \\
\hline pertain the selection hand of the GA on $\mathbf{X}_{\mathbf{I}}(\mathbf{t})$ \\
\hline division $\overline{\mathbf{X}}_{\mathbf{1}}(\mathbf{t})$ into part $_{\mathbf{n}}$ sub-partitions \\
\hline For $\left(\mathbf{i}=\mathbf{1}: \mathbf{i} \leq\right.$ part $\left._{\mathbf{n}}\right)$ do \\
\hline Apply the mathematical crossover on each sub-partition \\
\hline End for \\
\hline Apply the GA mutation operator on $\bar{X}_{\mathbf{I}}(\mathbf{t})$ \\
\hline Update the solutions \\
\hline Set $\mathbf{t}=\mathbf{t}+\mathbf{1}$ //iteration counter is increased \\
\hline Until $\left(\mathbf{t}>\mathbf{M a x}_{\mathbf{i t r}}\right) / /$ Termination criteria \\
\hline Display the best solution $\mathbf{P}_{\text {best }}$ \\
\hline End \\
\hline
\end{tabular}

The proposed HPSOGA algorithm starts by setting its parameter values. Then the iteration $t$ is set and the primary population is randomly generated and each solution in the population is evaluated. The PSO \& GA operations are 
recurring until break conditions are fulfilled. The new solutions are produced by pertaining the standard PSO on the whole population. Then a transitional population from the existing individual is selected by pertaining GA choice operator. In command to boost the variety of the exploration and prevail over the dimensionality problem, the existing population is partitioned into part $_{\mathrm{n}}$ sub-population where each subpopulation size is $\mathrm{v} \times \eta$. $\mathrm{v}$ is the number of variables in each partition and $\eta$ is the number of solutions in each partition. Then the mathematical intersect operator is pertained on each sub-population and the genetic mutation operator is pertained in the entire population consecutively to evade the premature convergence. Finally the solutions in the population are estimated by manipulating its fitness function. Then $t$ is rising and the overall processes are repeated until break conditions are fulfilled. Thus best solutions are obtained which can be employed for the adaptive filter.

Based on this hybrid algorithm, the adaptive filter can be designed with global optimization. For adaptive noise cancellation, HPSOGA's aim is to reduce the residual noise by identifying most favorable weight coefficients for the adaptive filter. For this purpose, a cost function is calculated by approximation of the mean square error (MSE) between the arriving samples $d[N]$ and the adaptive filter output $y[N]$. The cost function is given as:

$$
\mathrm{C}_{\mathrm{i}, \mathrm{k}}=\frac{1}{\mathrm{H}} \sum_{\mathrm{n}=1}^{\mathrm{H}} \mathrm{e}_{\mathrm{i}, \mathrm{k}}[\mathrm{n}]^{2}
$$

Where, $e_{i, k}[n]$ is the inaccuracy signal at $k^{\text {th }}$ iteration for $i^{\text {th }}$ element and $\mathrm{H}$ is the amount of input trials to the filter. The $\mathrm{y}[\mathrm{N}]$ is the outcome of updating filter parameters with the weight coefficients provided by HPSOGA to the adaptive filter. HPSOGA adjusts a set of particles and outlines each location as well as a preliminary speed as zero. The location vector characterizes the weight coefficients, set as $\mathrm{N}$ number of haphazard solutions. Using the position, principles of the cost function $\mathrm{C}_{\mathrm{i}, \mathrm{k}}$ are estimated for $\mathrm{N}$ particles and $\mathrm{k}$ iterations. Corresponding particle position for the minimum importance of cost function is set as $\mathrm{P}_{\text {best }}$ which is obtained by performing HPSOGA. Until the algorithm congregates to a global best possible solution or a determined iteration $\left(\operatorname{Max}_{i t r}\right)$, these procedures are recurring. Thus developed model of AF-SDSM-SMOR eliminates the noise elements adaptively. Then the opportunistic routing is performed as in SDS-M-SMOR by considering Sparsity aware distributed spectrum map. The PSNR and MSE parameters are useful in analyzing the behaviors' of the routing model under noisy environment.

\section{PERFORMANCE EVAlUATION}

The simulations of the proposed AF-SDS-M-SMOR routing model is evaluated and compared with the help of MATLAB tool. This routing model is evaluated with the help of delay, throughput, BER (Bit Error Rate), PSNR (Peak Signal-to-noise Ratio) and MSE. Performance of AF-SDS-MSMOR is compared with that of SMOR, M-SMOR and SDSM-SMOR routing models [15]. The comparisons are made between these routing models separately for regular and large scale networks.
Fig. 2 shows the end-to-end delay comparison. For both regular and large scale CRAHN the AF-SDS-M-SMOR model outperforms the other models with less delay. This is due to the fact that the noise elimination releases free spectrum which can be utilized for faster data transmission.

The improvement in the noise removal increases the throughput by increasing the spectrum access for the users which can be seen in Fig. 3. For both CRAHNs, the AF-SDSM-SMOR has higher throughput as it has a specialized noise cancellation block along with Sparsity aware routing.

BER is minimized when the noises are filtered and similarly the loss of data is also minimized. Fig. 4 shows the BER comparison which depicts that AF-SDS-M-SMOR has less BER compared to other models. This can be attributed to the introduction of global optimum solutions of the adaptive filter.
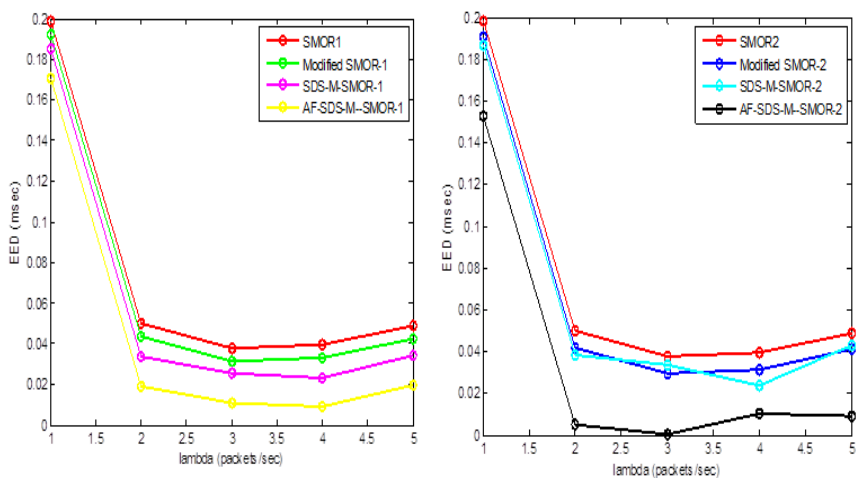

Fig. 2. End to end delay comparison a) Regular CRAHN, b) Large scale CRAHN
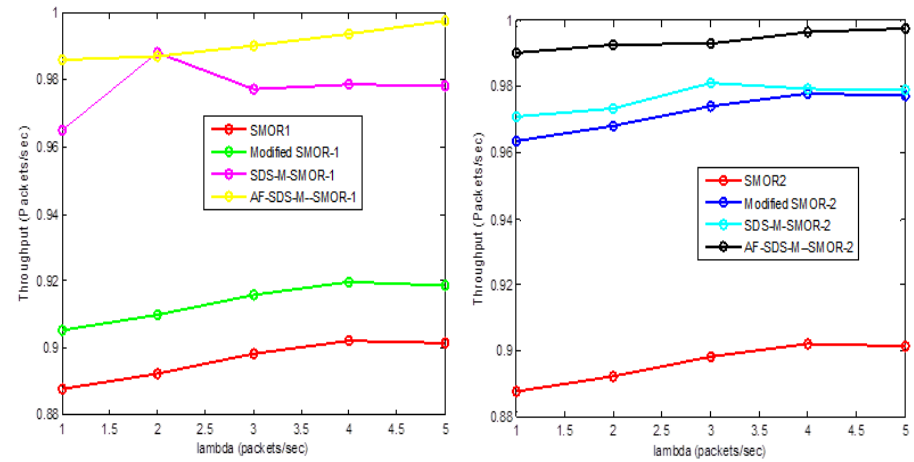

Fig. 3. Throughput comparison a) Regular CRAHN, b) Large scale CRAHN. 

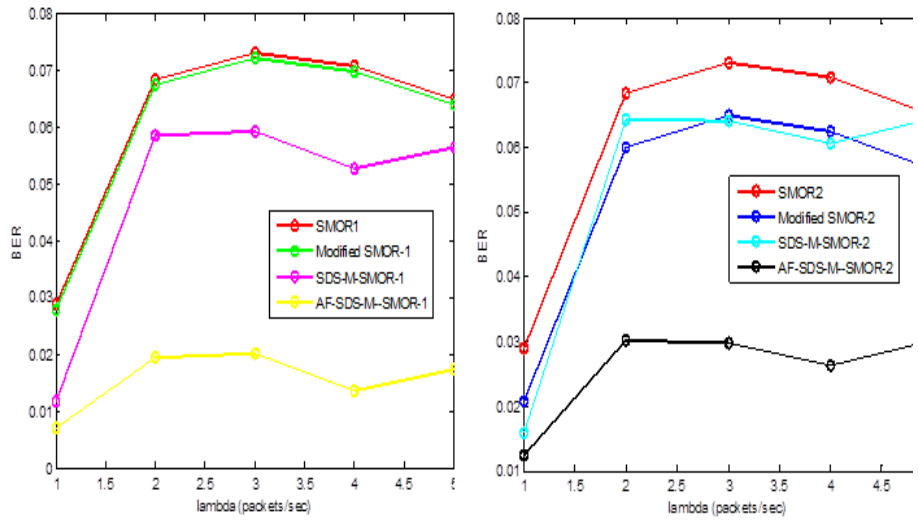

Fig. 4. BER comparison a) Regular CRAHN, b) Large scale CRAHN.
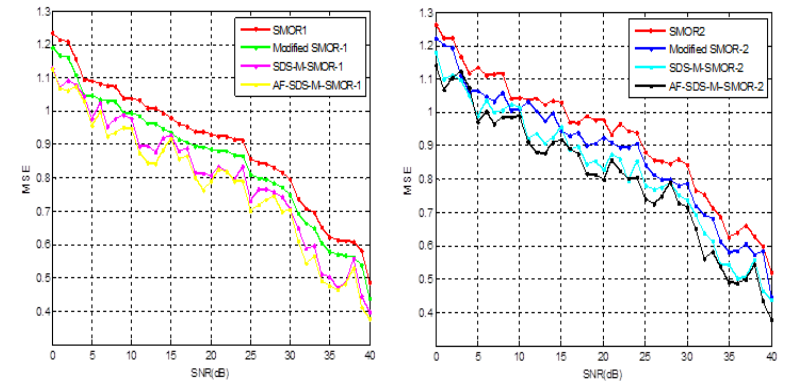

Fig. 5. MSE comparison a) Regular CRAHN, b) Large scale CRAHN.
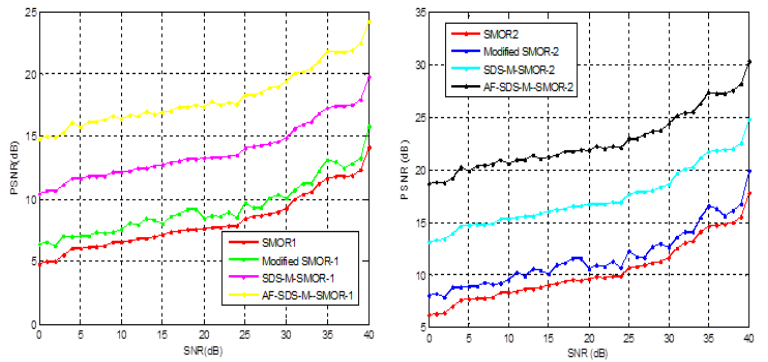

Fig. 6. PSNR comparison a) Regular CRAHN, b) Large scale CRAHN.

MSE and PSNR are most important parameters in evaluating the performance of the system with the effects of noise. Fig. 5 and 6 shows the MSE and PSNR comparisons respectively. AF-SDS-M-SMOR outshines the other systems. This is due to the Sparsity exploitation of the model reduces the error rate while the noise cancellation improves the PSNR. Thus reliable transmission is ensured through the proposed opportunistic routing model.

\section{CONCLUSION}

In this paper an efficient noise cancellation model has been developed using HPSOGA based adaptive filter which was incorporated with the Sparsity aware opportunistic routing model SDS-M-SMOR [15]. This concept of routing reduced the noise while also improving the overall throughput. The noise cancellation model significantly enhanced the opportunistic routing by providing efficient data transmission. The extensive evaluation of the proposed model has been compared with the other available routing model which proved that this research work can enhance the routing behavior of the CRAHN. In the future, interference avoidance and elimination of the error nodes will be investigated for better performance.

\section{REFERENCES}

[1] Sahai, A. (2005). Spectrum sensing: fundamental limits and practical challenges. IEEE International Symposium on New Frontiers in Dynamic Spectrum Access Networks (DySPAN '05), Baltimore, Md, USA.

[2] Zeng, Y., Liang, Y. C., Hoang, A. T., \& Zhang, R. (2010). A review on spectrum sensing for cognitive radio: challenges and solutions. EURASIP Journal on Advances in Signal Processing, 2010(1), 381465.

[3] Martinek, R., \& Zidek, J. (2010). Use of adaptive filtering for noise reduction in communications systems. In Applied Electronics (AE), 2010 International Conference on (pp. 1-6). IEEE.

[4] Kaabouch, N. (Ed.). (2014). Handbook of Research on SoftwareDefined and Cognitive Radio Technologies for Dynamic Spectrum Management. IGI Global.

[5] Liu, J., Zhang, H., Song, H., \& Mathew, G. (2012). U.S. Patent No. $8,295,001$. Washington, DC: U.S. Patent and Trademark Office.

[6] Haykin, S., \& Widrow, B. (Eds.). (2003). Least-mean-square adaptive filters(Vol. 31). John Wiley \& Sons.

[7] Mohammed, J. R. (2007). A new simple adaptive noise cancellation scheme based on ALE and NLMS filter. In Communication Networks and Services Research, 2007. CNSR'07. Fifth Annual Conference on (pp. 245-254). IEEE.

[8] Yim, K. H., Kim, J. B., Lee, T. P., \& Ahn, D. S. (1999). Genetic adaptive IIR filtering algorithm for active noise control. In Fuzzy Systems Conference Proceedings, 1999. FUZZ-IEEE'99. 1999 IEEE International (Vol. 3, pp. 1723-1728). IEEE.

[9] Yu, H., Zhu, H., \& Shi, Y. (2004). RBF networks trained by genetic algorithm appiled in active control of noise and vibration. Acoustical Science and Technology, 25(1), 109-111.

[10] Chang, C. Y., \& Chen, D. R. (2010). Active noise cancellation without secondary path identification by using an adaptive genetic algorithm. IEEE Transactions on Instrumentation and Measurement, 59(9), 2315-2327.

[11] Russo, F., \& Sicuranza, G. L. (2007). Accuracy and performance evaluation in the genetic optimization of nonlinear systems for active noise control. IEEE Transactions on Instrumentation and Measurement, 56(4), 1443-1450.

[12] George, N. V., \& Panda, G. (2012). A robust evolutionary feedforward active noise control system using Wilcoxon norm and particle swarm optimization algorithm. Expert Systems with Applications, 39(8), 75747580.

[13] Rout, N. K., Das, D. P., \& Panda, G. (2012). Particle swarm optimization based active noise control algorithm without secondary path identification. IEEE Transactions on Instrumentation and Measurement, 61(2), 554-563.

[14] Poli, R., Kennedy, J., \& Blackwell, T. (2007). Particle swarm optimization. Swarm intelligence, 1(1), 33-57.

[15] Hesham Mohammed Ali Abdullah, Dr.A.V. Senthil Kumar, Modified SMOR Using Sparsity Aware Distributed Spectrum Map for Enhanced Opportunistic Routing in Cognitive Radio Adhoc Networks. Journal of Advanced Research in Dynamical and Control Systems, (2017) (Vol. 9 (6), pp. 184-196). 